\section{Summary}

The coronary circulation was studied in two patients with proven sarcoidosis and myocardial defects at Thallium 201 scintigraphy, normal left ventricular function and coronary arteriograms. Coronary sinus blood flow was measured in the basal state, after reactive hyperemia induced by contrast medium and after intravenous dipyridamole. Basal coronary sinus blood flow was below values obtained in eight normal subjects and coronary resistance was increased.

Reactive hyperemia resulted in a 3.09 and a 2.56 fold increase in coronary sinus blood flow in the two patients, respectively, compared to a $1.99 \pm 0.24$ fold increase in normals. The contrast medium/basal coronary resistance ratio was 0.32 and 0.39 in the two patients, respectively, and $0.48 \pm 0.06$ in normals.

In the second patient, an i.v. dipyridamole infusion (0.14 $\mathrm{mg}$. $\left.\mathrm{kg}^{-1} \cdot \mathrm{min}^{-1} \times 4 \mathrm{~min}\right)$ resulted in values of coronary sinus blood flow and coronary resistance within the normal range. In this patient, the Thallium $20 \mathrm{I}$ abnormalities disappeared af ter i.v. dipyridamole as well as after a two week treatment by oral dipyridamole (450 mg per day). These results suggest the existence of a reversible microvascular spasm in cardiac sarcoidosis. This could explain, in part, the resting angina pectoris and Thallium $20 \mathrm{I}$ scan abnormalities. It also could be involved in a process which leads to the dilated cardiomyopathy observed in such patients. Finally, dipyridamole could represent a new therapeutic approach of cardiac sarcoidosis.

\title{
Sarcoïdose cardiaque et vasoconstriction chronique \\ de la microcirculation coronaire
}

\author{
Alain Nitenberg, Jean-Marc Foult, Isabelle Antony \\ Dominique Valeyre, Jeannine Perrenec \\ Philippe Tellier, Françoise Blanchet
}

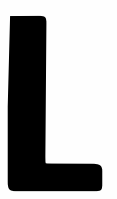

'existence d'une vasoconstriction réversible de la microcirculation coronaire a été démontrée dans la cardiomyopathie du hamster Syrien [I]. Chez l'homme, une telle éventualité a été évoquée pour expliquer les atteintes myocardiques de la sclérodermie [2]. Les études anatomopathologiques du cœur sarcoïdien ont non seulement révélé des localisations granulomateuses [3-7] mais aussi artériolaires de la maladie $[5,8]$, ainsi que quelques altérations ultrastructurales des cellules endothéliales capillaires [9]. En revanche, on n'a jamais décrit de lésions des gros troncs coronaires $[5,6]$. La présence de ces altérations artériolaires et capillaires pourrait entraîner des troubles de la perfusion myocardique dans la sarcoïdose cardiaque. Par ailleurs, plusieurs auteurs ont mentionné la survenue de douleurs angineuses de repos chez certains malades atteints de sarcoïdose [10-I2]. Ce travail, réalisé chez deux patients atteints de sarcoïdose sans anomalie de la fonction ventriculaire gauche, ni de lésion des gros troncs coronaires apporte des arguments en faveur de l'existence d'une vasoconstriction réversible de la microcirculation coronaire.

\section{Patients}

Le premier sujet est un homme de 38 ans, d'origine maghrébine, dont la sarcoïdose est découverte en 1974 à la suite d'une splénectomie pour splénomégalie avec hypersplénisme. En I980, l'électrocardiogramme révèle un bloc complet de la branche droite et, en I982, apparaît un angor de repos typique sans douleur d'effort. L'image radiologique du thorax et l'échocardiographie bidimensionnelle sont normales ainsi que glycémie, lipidémie et calcémie; la concentration sérique de l'enzyme de conversion est de $47 \mathrm{U} / \mathrm{ml}$ (normale $40 \pm 20 \mathrm{U} / \mathrm{ml}$ ). La scintigraphie myocardique au Gallium 67 ne montre pas de fixation anormale. Le second sujet est un homme de 36 ans, d'ethnie caucasienne, dont la sarcoïdose est diagnostiquée en mai 1984 à propos d'un tableau clinique comportant une dyspnée, une fièvre, un amaigrissement et des 
douleurs angineuses de repos. L'électrocardiogrammé est alors normal, l'image radiologique du thorax montre une miliaire pulmonaire et un cœur de volume normal. Le diagnostic est établi sur la présence de granulomes dans la biopsie bronchique. Glycémie, lipidémie, calcémie sont normales, la concentration sérique de l'enzyme de conversion est de $280 \mathrm{U} / \mathrm{ml}$. La scintigraphie myocardique au Gallium 67 est tout à fait normale.

\section{Méthode du cathétérisme cardiaque}

Chez ces patients, à jeun depuis au moins douze heures, aucune prémédication n'a été administrée. Après anesthésie locale, un double micromanomètre $7 \mathrm{~F}$ (Millar PC-770) a été placé dans le ventricule gauche par voie fémorale afin de recueillir simultanément les pressions dans le ventricule gauche et l'acrte. Un désilet $7 \mathrm{~F}$ a été placé dans l'autre artère fémorale pour permettre la réalisation de la ventriculographie gauche et de la coronarographie. Un cathéter de thermodilution (Swan Ganz ${ }_{7} F$ ) a été placé dans l'artère pulmonaire par voie veineuse fémorale afin de mesurer le débit cardiaque. Enfin, un cathéter de thermodilution coronaire (Wilton Webster) a été mis en place dans le sinus coronaire par voie sous-clavière gauche afin de mesurer le débit du sinus coronaire [13]; la position de cathéter a été contrôlée régulièrement avant chaque mesure de débit afin que la thermistance proximale de ce cathéter soit située face à l'arrivée de la veine irterventriculaire postérieure dans le sinus coronaire [I4].

\begin{tabular}{|c|c|c|c|c|c|}
\hline & $\begin{array}{r}\text { RÉSULT } \\
\text { (moyennes } \pm\end{array}$ & $\begin{array}{l}\text { Tableau } \\
\text { TS HÉMOD } \\
\text { DS pour les }\end{array}$ & $\begin{array}{l}\text { NAMIQUES } \\
\text { sujets normau }\end{array}$ & & \\
\hline & sujets nor & aux $(n=8)$ & Patient $n^{\circ} 1$ & Patic & $n^{\circ} 2$ \\
\hline & B & D & B & B & D \\
\hline & $84 \pm 14$ & $106 \pm 20$ & 70 & 75 & 96 \\
\hline IC & $3,5 \pm 0,4$ & $4,9 \pm 0,7$ & 3,5 & 3,4 & 5,2 \\
\hline PSVG & $133 \pm 16$ & $121 \pm 14$ & 108 & 146 & 130 \\
\hline PTDVG & $8 \pm 2$ & $7 \pm 4$ & 8 & 10 & 10 \\
\hline PMDVG & $5 \pm 2$ & $4 \pm 4$ & 5 & 4 & 6 \\
\hline $\begin{array}{l}\mathrm{mmHHg} \\
\mathrm{POD}\end{array}$ & $3 \pm 2$ & $2 \pm 2$ & 0 & 6 & 2 \\
\hline PAo & $101 \pm 9$ & $92 \pm 10$ & 88 & 118 & 119 \\
\hline RVS & $18 \pm 2$ & $12 \pm 2$ & 16 & 17 & 12 \\
\hline $\begin{array}{l}\text { PAP } \\
\mathrm{mmHg}\end{array}$ & $13 \pm 4$ & $12 \pm 5$ & 11 & 18 & 16 \\
\hline
\end{tabular}

Abréviations : $B$ : état basal; $D$ : dipyridamole; $F C$ : fréquence cardiaque; IC index cardiaque; PSVG : pression systolique du ventricule gauche; PTDVG : pression télédiastolique du ventricule gauche; PMDVG : pression diastolique moyenne du ventricule gauche; $P O D$ : pression moyenne de l'oreillette droite; $P A o$ : pression aortique moyenne; RVS : résistances vasculaires systémiques; $P A P$ : pression moyenne de l'artère pulmonaire.
Après la ventriculographie gauche et la coronarographie, un délai de trente minutes a été observé, afin que les effets du produit de contraste soient éliminés. Les pressions ventriculaire gauche, aortique et auriculaire droite, ainsi que les débits cardiaque et du sinus coronaire ont été mesurés à l'état basal. Des prélèvements de sang artériel, de l'artère pulmonaire et du sinus coronaire, ont été recueillis afin d'étudier les gaz du sang et l'extraction de lactate chez le patient $n^{\circ} 2$.

Chez les deux malades, une réaction hyperémique a été induite par l'injection de $8 \mathrm{ml}$ de produit de contraste (ioxaglate meglumine, 580 mosm. $\left.\mathrm{kg}^{-1}\right)$ dans l'artère coronaire gauche $[15,16]$ en même temps qu'ont été enregistrés de façon continue les pressions ventriculaire gauche et aortique, ainsi que le débit du sinus coronaire. Chez le patient $\mathrm{n}^{\circ} 2$, deux minutes après que le débit coronaire soit revenu à sa valeur basale, le même protocole a été répété cinq minutes après la fin d'une perfusion de $0,14 \mathrm{mg}$. $\mathrm{kg}^{-1} \cdot \min ^{-1}$ de dipyridamole pendant quatre minutes [17-19].

Huit patients, ne présentant pas de douleur thoracique, non traités médicalement et devant subir un cathétérisme cardiaque du fait d'une cardiopathie existante susceptible d'être traitée chirurgicalement, ont été pris comme sujets témoins. Il s'agit de six femmes et de deux hommes (âge moyen $46 \pm$ I I ans) : cinq sujets sont porteurs d'une sténose mitrale modérée (débit cardiaque normal et pression pulmonaire bloquée inférieure à $15 \mathrm{mmHg}$ ), un sujet est porteur d'une sténose pulmonaire modérée (pression systolique du ventricule droit : $45 \mathrm{mmHg}$ ), un sujet est atteint de maladie de Roger $\left({ }^{1}\right)$ et le dernier sujet présente un patent foramen ovale $\left({ }^{2}\right)$. Ces deux derniers patients n'ont pas de shunt gauche-droite prouvé par l'oxymétrie. Tous ces sujets ont une fonction ventriculaire gauche normale et une artério-

(1) Communication interventriculaire sans shunt détectable par oxymétrie.

( $\left.{ }^{2}\right)$ Persistance de la perméabilité du trou de Botal sans shunt gauche-droite. 
graphie coronaire normale. Une réaction hypérémique au produit de contraste et une perfusion de dipyridamole ont été réalisées chez l'ensemble de ces sujets.

Une biopsie endomyocardique du ventricule gauche [20] a été faite chez ces deux malades et examinée en microscopie optique.

\section{Scintigraphie myocardique}

Une scintigraphie myocardique de perfusion au Thallium 20I a été effectuée, au repos chez le patient $\mathrm{n}^{\circ} \mathrm{I}$, au repos et après injection intraveineuse de dipyridamole chez le patient $n^{\circ} 2$. Les scintigraphies sont obtenues après injection intraveineuse de $74 \mathrm{MBq}$ de chlorure de Thallium 201. Chaque vue est enregistrée par une gamma-caméra interfacée avec un système informatique (Informatek SIMIS 3) qui recueille 600000 à 700000 points par vie. Les images sont traitées par la technique de soustraction de Goris et coll. [2]. Le seuil d'hypofixation est fixé à $25 \%$ au-dessous de la zone de fixation maximum du Tha!lium par unité de surface.

\section{Résultats}

L'exploration hémodynamique de ces deux patients fournit des valeurs qui sont situées dans l'intervalle de confiance (moyenne $\pm 2 \mathrm{DS}$ ) de huit sujets normaux (tableau I). La fonction ventriculaire gauche est dans les limites de la normale (tableau II). Il existe une augmentation modérée du volume télédiastolique ventriculaire gauche chez le patient $\mathrm{n}^{\circ} \mathrm{I}$ (tableau II). La coronarographie de ces deux patients met en évidence des artères coronaires dilatées, souples et régulières et un ralentissement circulatoire du produit de contraste.

Chez les deux patients à l'état basal, le débit du sinus coronaire est faible et la résistance coronaire est élevée. La réaction hyperémique au produit de contraste est ample et prolongée (tableau III, page suivante et figure I) : le volume de l'hyperémie, la durée de l'hyperémie, le rapport entre le pic d'hyperémie et le débit basal et l'amplitude de la chute de la résistance coronaire pendant l'hype-
Tableau II

RÉSULTATS ANGIOGRAPHIQUES

(moyennes \pm DS pour les sujets normaux)

\begin{tabular}{|l|c|c|c|}
\hline & $\begin{array}{c}\text { sujets } \\
\text { normaux }(n=8)\end{array}$ & Patient $n^{\circ} 1$ & patient $n^{\circ} 2$ \\
\hline $\mathrm{VTD}$ & $76 \pm 15$ & 118 & 77 \\
$\mathrm{ml} \cdot \mathrm{m}^{-2}$ & $64 \pm 11$ & 82 & 69 \\
$\mathrm{MVG}$ & $61 \pm 4$ & 64 & 67 \\
$\mathrm{~g} \cdot \mathrm{m}^{-2}$ & $4,80 \pm 0,83$ & 4,17 & 4,12 \\
$\mathrm{FE}$ & & & \\
\hline $\mathrm{\sigma} T S / \mathrm{VTS}$ & $\mathrm{g} \cdot \mathrm{cm}^{-2} \cdot \mathrm{ml}^{-1}$ & & \\
\hline
\end{tabular}

Abréviations : VTD : volume télédiastolique du ventricule gauche; MVG : masse myocardique du ventricule gauche [22]; FE: fraction d'éjection; $\sigma$ TS/VTS : rapport contrainte/volume télésystolique [23, 24].

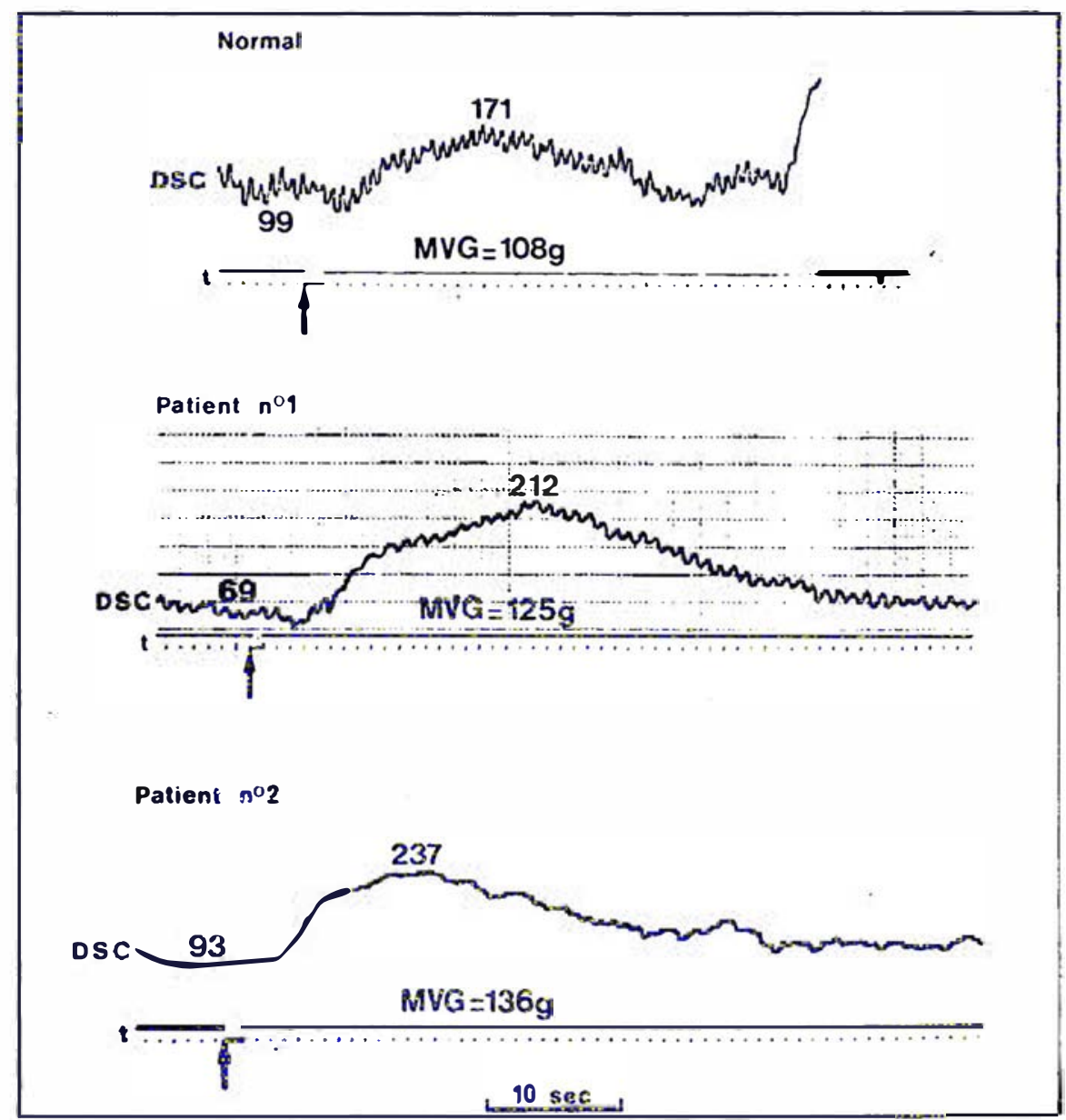

Figure 1. Aspect de la réaction hyperémigtys au produit de contraste chaz un sijjet normai et choz los diux parients. Noter la faible valeur du débit sanguin du sinus coronaire (OSC: $\mathrm{ml}, \mathrm{min}^{-1}$ ) avant l'injection du produit de coritrasîe (indiguée pas ies llècines) et l'importance de la réaction hiperérnique. MVG: masse myocardique du veniricule gauche; $t$ : temps (1 top pas secunde). 


\begin{tabular}{|c|c|c|c|c|c|c|}
\hline \multicolumn{7}{|c|}{$\begin{array}{l}\text { Tableau III } \\
\text { ORONAIRE AU PRODUIT DE CONTRASTE } \\
\text { S pour les sujets normaux) }\end{array}$} \\
\hline & \multicolumn{2}{|c|}{ sujets normaux $(n=8)$} & \multicolumn{2}{|c|}{ patient $n^{\circ} 1$} & \multicolumn{2}{|c|}{ patient $n^{\circ} 2$} \\
\hline & B & RHC & B & RHC & B & $\mathrm{RHC}$ \\
\hline $\begin{array}{l}\text { DSC } \\
\mathrm{ml} .100 \mathrm{~g}^{-1} \cdot \mathrm{min}^{-1} \\
\mathrm{RC} \\
\mathrm{mmHg} \cdot \mathrm{ml}^{-1} \cdot 100 \mathrm{~g} \cdot \mathrm{min}\end{array}$ & $\begin{array}{c}103 \pm 16 \\
0,94 \pm 0,10\end{array}$ & $\begin{array}{c}203 \pm 33 \\
0,48 \pm 0,06\end{array}$ & 55 & $\begin{array}{l}170 \\
0,49\end{array}$ & 68 & $\begin{array}{r}174 \\
0,66\end{array}$ \\
\hline $\begin{array}{l}\mathrm{RHCt} \\
\mathrm{seC} \\
\Delta \mathrm{Q} \\
\mathrm{ml} .100 \mathrm{~g}^{-1} \\
\mathrm{DSC}_{\mathrm{RHC} / \mathrm{B}} \\
\mathrm{RC}_{\mathrm{RHC} / \mathrm{B}}\end{array}$ & \multicolumn{2}{|c|}{$\begin{array}{l}26,1 \pm 2,7 \\
18,4 \pm 2,6 \\
1,99 \pm 0,24 \\
0,48 \pm 0,06\end{array}$} & \multicolumn{2}{|c|}{$\begin{array}{r}42,0 \\
27,9 \\
\\
3,09 \\
0,32\end{array}$} & \multicolumn{2}{|c|}{$\begin{array}{r}41,6 \\
28,6 \\
2,56 \\
0,39\end{array}$} \\
\hline
\end{tabular}

Abréviations : $B$ : état basal; RHC : réaction hyperémique coronaire; $D S C$ : débit sanguin du sinus coronaire; RC: résistance coronaire (pression aortique moyenne moins pression diastolique moyenne du ventricule gauche/débit sanguin du sinus coronaire); $R H C t$ : durée de la réaction hyperémique coronaire; $\triangle Q$ : volume de l'hyperémie (débit coronaire moyen pendant l'hyperémie moins débit coronaire basal $\times$ durée de l'hyperémie).

rểmie sont nettement supểrieurs à ce qui est observé chez les sujets normaux, alors que le pic de débit est situé dans les limites des valeurs normales. Après injection intraveineuse de dipyridamole chez le patient $n^{\circ} 2$ le débit et la résistance coronaires sont comparables à ceux des sujets normaux (tableau $I V$ ), faisant apparaître une amplitude d'augmentation du débit coronaire et de chute de la résistance coronaire nettement plus élevées que chez des sujets normaux. D'autre part, les valeurs de la pression partielle en $\mathrm{O}_{2}$ et du contenu en $\mathrm{O}_{2}$ du sang veineux coronaire sont faibles, la différence artério-veineuse coronaire en $\mathrm{O}_{2}$ est élevée à l'état basal. Toutes ces valeurs sont situées dans les limites de la normale après dipyridamole. Enfin, on note une augmentation de l'extraction de lactate, initialement faible chez ce patient (tableau IV).

$\mathrm{La}$ biopsie endomyocardique du ventricule gauche montre chez le sujet $\mathrm{n}^{\circ} \mathrm{I}$, en microscopie optique (figure 2), l'existence de deux granulomes et de quelques zones de fibrose sous-endocardique alors que les cellules myocardiques sont

\begin{tabular}{|c|c|c|c|c|}
\hline \multicolumn{5}{|c|}{$\begin{array}{l}\text { Tableau IV } \\
\text { LA CIRCULATION CORONAIRE } \\
\text { MYOCARDE PAR LE DIPYRIDAMOLE } \\
\text { S pour les sujets normaux) }\end{array}$} \\
\hline & \multicolumn{2}{|c|}{ sujets normaux $(n=8)$} & \multicolumn{2}{|c|}{ patient $n^{\circ} 2$} \\
\hline & B & D & B & D \\
\hline $\begin{array}{l}\text { DSC } \\
\mathrm{ml} .100 \mathrm{~g}^{-1} \cdot \mathrm{min}^{-1} \\
\mathrm{RC} \\
\mathrm{mmHg} \cdot \mathrm{ml}^{-1} \cdot 100 \mathrm{~g} \cdot \mathrm{min} \\
\mathrm{a}-\mathrm{SCO}_{2} \mathrm{D} \\
\mathrm{ml} .100 \mathrm{ml}^{-1} \\
\mathrm{O}_{2} \mathrm{SC} \\
\mathrm{ml} .100 \mathrm{ml}^{-1} \\
\mathrm{PO}_{2} \mathrm{SC} \\
\mathrm{kPa} \\
\mathrm{MV} \mathrm{O}_{2} \\
\mathrm{ml} .100 \mathrm{~g}^{-1} \cdot \text { min }^{-1} \\
\text { Extraction de lactate } \\
0\end{array}$ & $\begin{array}{c}103 \pm 16 \\
0,94 \pm 0,10 \\
12,6 \pm 0,9 \\
4,7 \pm 0,3 \\
2,74 \pm 0,38 \\
13,1 \pm 2,1 \\
61 \pm 13\end{array}$ & $\begin{array}{c}411 \pm 64 \\
0,22 \pm 0,03 \\
3,6 \pm 0,4 \\
12,8 \pm 0,4 \\
5,62 \pm 0,50 \\
14,7 \pm 2,6 \\
56 \pm 21\end{array}$ & $\begin{array}{c}68 \\
1,68 \\
15,9 \\
4,1 \\
1,90 \\
10,8 \\
7\end{array}$ & $\begin{array}{r}474 \\
0,24 \\
3,9 \\
15,3 \\
4,90 \\
18,5 \\
41\end{array}$ \\
\hline $\begin{array}{l}\mathrm{DSC} D / 8 \\
\mathrm{RC} \mathrm{C}_{\mathrm{D} / \mathrm{B}}\end{array}$ & \multicolumn{2}{|c|}{$\begin{array}{l}4,02 \pm 0,52 \\
0,23 \pm 0,04\end{array}$} & \multicolumn{2}{|c|}{$\begin{array}{l}6,97 \\
0,14\end{array}$} \\
\hline
\end{tabular}

Abréviations : $B$ : état basal; $D$ : dipyridamole; $D S C$ : débit sanguin du sinus coronaire; $R C$ : résistance coronaire (pression aortique moyenne-pression diastolique moyenne du ventricule gauche]/débit du sinus coronaire); a- $\mathrm{SCO}_{2} \mathrm{D}$ : différence artério-veineuse coronaire en oxygène; $\mathrm{O}_{2} S C$ : contenu en oxygène du sang du sinus coronaire; $\mathrm{PO}_{2} \mathrm{SC}$ : pression partielle en oxygène du sang du sinus coronaire; $\mathrm{MVO}_{2}$ : consommation d'oxygène du myocarde. 


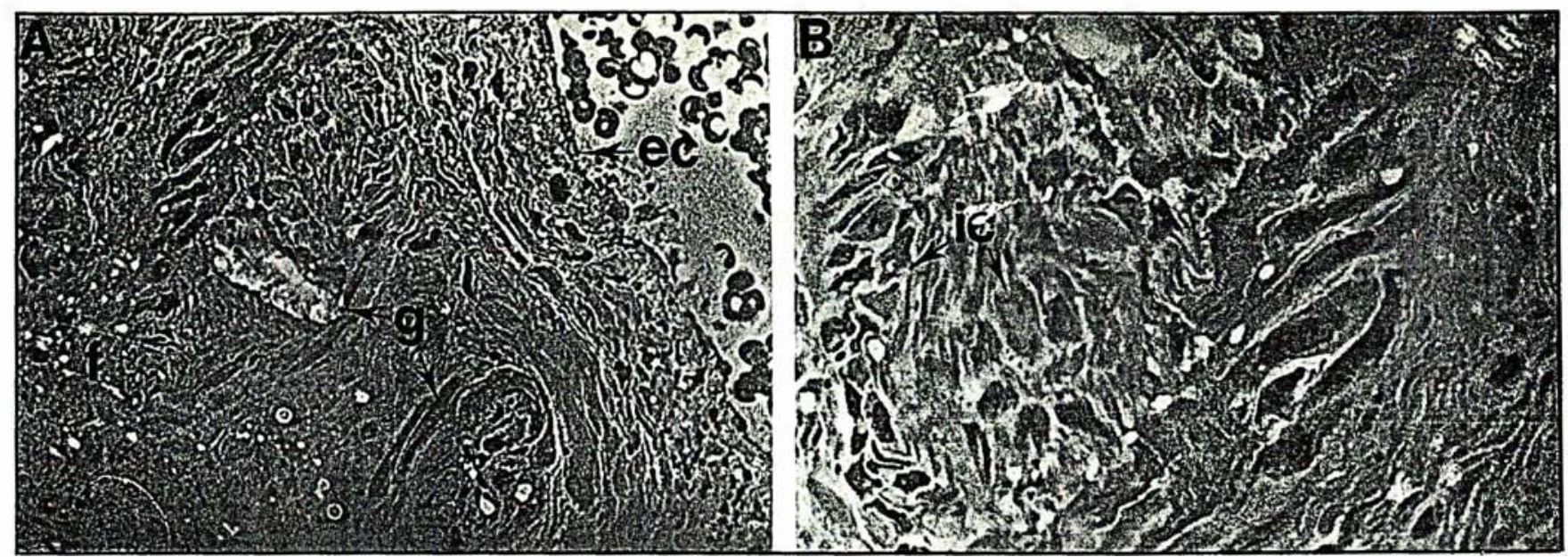

Figure 2. Biopsie endomyocardique du patient $n^{\circ} 1$ (microscopie optique). $A(\times 400)$ : fibrose (f), granulomes (g) et cellules endothéliales $(e c) . B(\times 800)$ : vue détaillée d'un granulome avec des cellules interstitielles (ic).

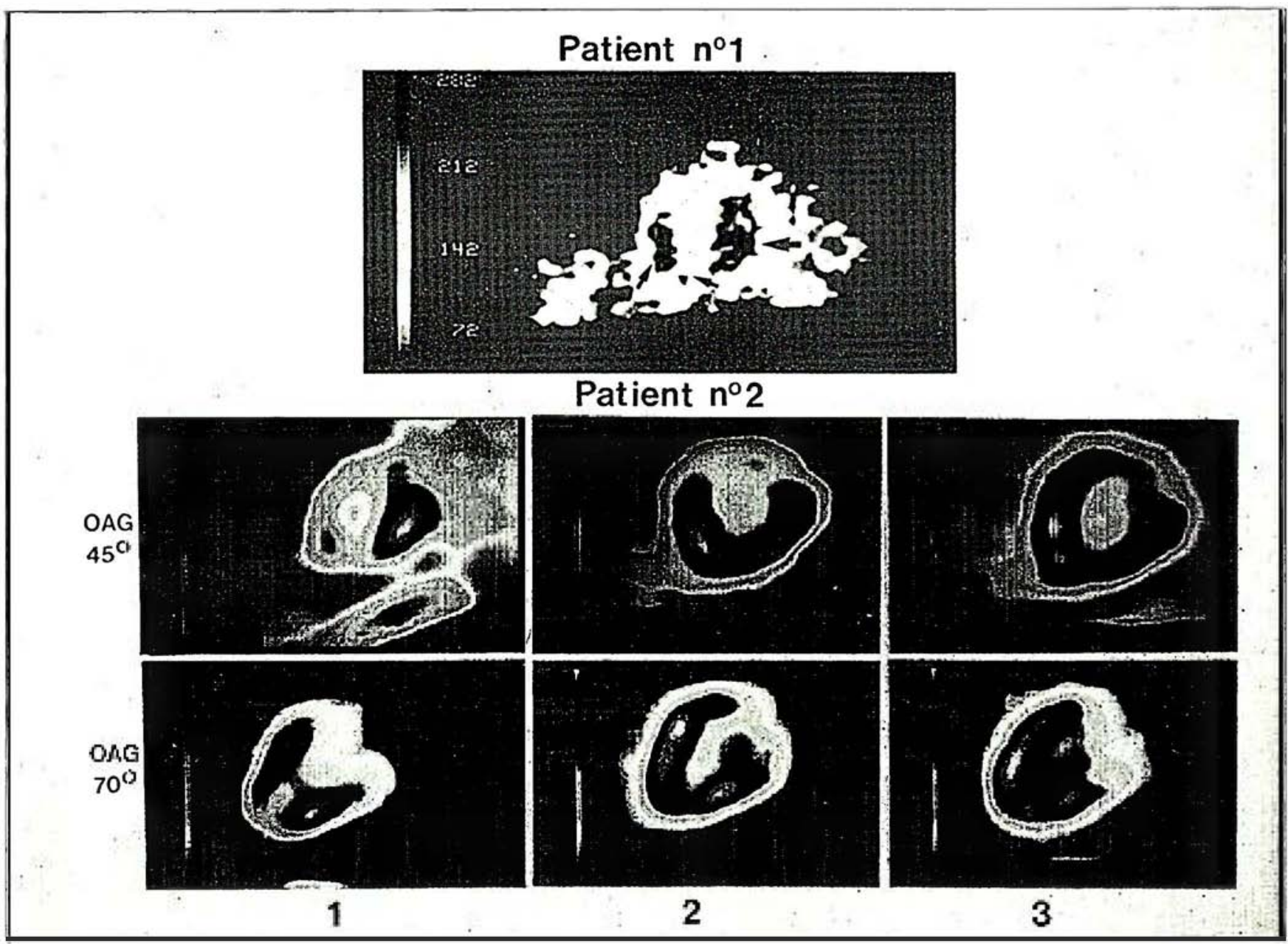

Figure 3. Scintigraphie myocardique de perfusion au Thallium 201. Chez le patient $n^{\circ} 1$ (OAG $\left.45^{\circ}\right)$, les petites flèches indiquent la faible activité de l'apex et du septum interventriculaire par rapport à celle de la paroi postérieure indiquée par la grande flèche. Le déficit de fixation apicale est large et profond. Chez le patient $n^{\circ}$ 2, les anomalies de la perfusion notées à l'état basal (1) disparaissent après injection intraveineuse de dipyridamole (2) et 15 jours après un traitement par le dipyridamole per os (3) (OAG : oblique antérieure gauche). 
normales. Chez le sujet $n^{\circ} 2$, les images observées sont normales. La scintigraphie myocardique de perfusion au Thallium 20I au repos montre une fixation hétérogène chez les deux patients (figure 3 , p. précédente). Le scintigramme devient normal chez le patient $\mathrm{n}^{\circ} 2$ après injection intraveineuse de dipyridamole (à la même dose que lors de l'étude hémodynamique) et après deux semaines de traitement par voie orale $(450 \mathrm{mg} / 24$ heures) (figure 3, p. précédente).

Ces observations suggèrent l'existence d'une vasoconstriction chronique et réversible de la microcirculation coronaire chez des sujets qui satisfont aux critères diagnostiques de sarcoïdose cardiaque proposés par Fleming [25]. Cette vasoconstriction chronique de la microcirculation coronaire qui pourrait être le résultat d'une libération locale de substances vasoactives (sérotonine et/ou histamine) par les macrophages ou les mastocytes qui entourent les granulomes [9], suppose l'existence d'une dissémination myocardique suffisante des granulomes [8]. Cette vasoconstriction peut expliquer la réversibilité des anomalies de la scintigraphie myocardique au Thallium observée spontanément dans certains cas [26], réversibilité incompatible avec une fibrose myocardique ou l'existence de granulomes confluents $[15,26]$. La normalisation du scintigramme au Thallium 20I par le dipyridamole intraveineux et après deux semaines de traitement per os chez le second sujet, constitue un argument de valeur en faveur de l'existence d'une telle vasoconstriction chronique [I].

L'existence d'une vasoconstriction réversible de la microcirculation coronaire chez certains sujets atteints de sarcoïdose cardiaque pourrait avoir d'importantes implications : elle pourrait expliquer l'origine des douleurs angineuses de repos signalées par certains patients [IO, I I, I5]; elle pourrait également expliquer l'évolution vers la myocardiopathie dilatée rencontrée dans certaines sarcoïdoses. Enfin, le dipyridamole pourrait constituer une nouvelle approche thérapeutique de la sarcoïdose cardiaque

\section{REFERENCES}

I. Factor SM, Minase T, Cho S, Dominitz R, Sonnenblick EH. Microvascular spasm in the cardiomyopathic Syrian hamster: a preventable cause of focal myocardial necrosis. Circulation 1982; 66: 342-54

2. Follansbee WP, Curtiss EI, Medsger TA, et al. Physiologic abnormalities of cardiac function in progressive systemic sclerosis with diffuse scleroderma. N Engl 7 Med 1984; 310: 142-8.

3. Bernstein M, Konzolmann FW, Sidlick DM. Boeck's sarcoid. Report of case with visceral involvement. Arch Intern Med 1929; 44: 721-34.

4. Fleming HA, Sarcoid heart disease. Br Heart 7 1974; 36: 54-68.

5. Matzui Y, Iwai K, Tachibana T, et al. Clinicopathological study on fatal myocardial sarcoidosis. Ann NY Acad Sci 1976; 278: 455-69.

6. Roberts WC, McAllister HA Jr, Ferrans VJ Sarcoidosis of the heart. A clinicopathologic study of 35 necropsy patients (Group I) and review of 78 previously described necropsy patients (Group II). Am 7 Med 1977; 63: 86-1 08.

7. Silverman KJ, Hutchins GM, Bulkley BN Cardiac sarcoid: a clinicopathologic study of 84 unsclected patients with systemic sarcoidosis. Circulation 1978; 58: I 204-I 1 .

8. James TN. De subitaneis Mortibus. XXV. Sarcoid heart disease. Circulation 1977; 56: 320-6.

9. Ferrans VJ, Hibbs RG, Black WC, Walsh JJ, Burch GE. Myocardial degeneration in cardiac sarcoidosis. Histochemical and electron microscopic studics. Am Heart 7 1965; 69: 159-72.

Io. Fox HB, Israel HL. Myocardial sarcoidosis: a cause of precordial pain. Clin Respir Phys (abstr) 1981; $17: 82 \mathrm{p}$

II. Kinney L, Jackson GL, Recve WC, Zclis R, Beers $E$. Thallium-scan myocardial defects and echocardiographic abnormalities in patients with sarcoidosis without clinical cardiac dysfunction. Am 7 Med 1980; 68: 497-503.

12. Valeyre D, Marsac J, Gambini G, et al. Assessment of sarcoidosis heart disease: a study of 37 patients with thoracic sarcoidosis. In Chretien J, Marsac J, Saltiel JC eds. Sarcoidosis and other granulomatous disorders. Paris: Pergamon Press, 1981: 296-300.

13. Ganz W, Tamura K, Marcus HS, Donoso R, Yoshida S, Swan HJC. Mcasurement of coronary sinus blood flow by continuous thermodilution in man. Circulation 1971; 44: 181-94.

14. Hood WB Jr. Regional venous drainage of the human heart. Br Heart 7 1968; 30: 105-9.

15. Mishima M, Inoue M, Hori M, et al. Validity of contrast hyperemia for clinical assessment of coronary flow reserve: the optimal dose of contrast medium and reproductibility of the technique. Cashet Cardiovasc Diagn 1983; 9: 55368.
16. Foult JM, Nitenberg A, Blanchet F, Zouiouêche $S$, Huyghebaert MF. Alterations in contrast medium induced coronary reactive hyperemia after bepridil in patients with coronary artery disease. Am Heart 7 1985; 109: 244-51.

17. Nitenberg A, Foult JM, Blanchet F, Zouiouèche S. Multifactorial determinants of reduced coronary flow reserve after dipyridamole in dilated cardiomyopathy. $\mathrm{Am} 7$ Cardiol 1985 ; 55: 748-54.

I8. Gould KL. Assessment of coronary stenosis with myocardial perfusion imaging during pharmacologic coronary vasodilation. IV. Limits of detection of stenosis with idealized experimental cross-sectional myocardial imaging. $\mathrm{Am} 7 \mathrm{Car}$ diol 1978; 42: 761-8.

19. Hoff man JIE. Maximal coronary flow and the concept of coronary vascular reserve. Circulation 1984; 70: 153-9.

20. Richardson PJ. King's endomyocardial bioptome. Lancet 1974; 1: 660-I.

21. Goris ML, Daspit SG, McLaughlin P. Interpolative background subtraction. I Nucl Med 1978; 19: 744-7.

22. Trenouth RS, Phelps NC, Neill WA Determinants of left ventricular hypertrophy and oxygen supply in chronic aortic valve discase. Circulation $1976 ; 53$ : 644-50.

23. Falsetti HL, Mates RE, Grant C, Greene DG Bunnell IL. Left ventricular wall stress calculated from one plane cineangiography: an approach to force velocity analysis in man. Circ Res 1970; 26 : $71-83$

24. Carabello BA, Nolan SP, McGuire LB. Assessment of preoperative left ventricular function in patients with mitral regurgitation: value of the end systolic wall stress-end systolic volume ratio. Circulation 1981; 64: 1212-17.

25. Fleming HA. Sarcoid heart disease: review and appeal. Thorax 1980; 35: 641-3.

26. Makler PT, Lavine SJ, Denenberg BS, Bove $A A$, Idell $S$. Redistribution on the thallium-scan in myocardial sarcoidosis: concise communication. 7 Nucl Med 1981; 22: 428-32.

\section{ADRESSES}

A. Nitenberg, J.-M. Foult, F. Blanchet : Service de physiologie et d'explorations fonctionnelles, Inserm U251, hôpital Bichat, Université ParisVII, 75877 Paris Cedex 18.

D. Valeyre : Service de pneumologie, hôpital Avicenne, 93009 Bobigny.

J. Perrenec : Unité de recherche cardiovasculaire, Inserm U2, hòpital Léon Bernard, 94450 LimeilBrévannes.

Ph. Tellier : Service de médecine nucléaire, hôpital Bichat, 75877 Paris Cedex 18.

\section{TIRES A PART}

A. Nitenberg : CHU Xavier Bichat, $75^{8} 77$ Paris Cedex 18. 